\title{
Pay for a Sliding Bloom Filter and Get Counting, Distinct Elements, and Entropy for Free
}

\author{
Eran Assaf \\ Hebrew University
}

\author{
Ran Ben Basat \\ Technion
}

\author{
Gil Einziger \\ Nokia Bell Labs
}

\author{
Roy Friedman \\ Technion
}

\begin{abstract}
For many networking applications, recent data is more significant than older data, motivating the need for sliding window solutions. Various capabilities, such as DDoS detection and load balancing, require insights about multiple metrics including Bloom filters, per-flow counting, count distinct and entropy estimation.

In this work, we present a unified construction that solves all the above problems in the sliding window model. Our single solution offers a better space to accuracy tradeoff than the state-of-the-art for each of these individual problems! We show this both analytically and by running multiple real Internet backbone and datacenter packet traces.
\end{abstract}

\section{Introduction}

Network measurements are at the core of many applications, such as load balancing, quality of service, anomaly/intrusion detection, and caching $[1,13,16,24,31]$. Measurement algorithms are required to cope with the throughput demands of modern links, forcing them to rely on scarcely available fast SRAM memory. However, such memory is limited in size [34], which motivates approximate solutions that conserve space.

Network algorithms often find recent data useful. For example, anomaly detection systems attempt to detect manifesting anomalies and a load balancer needs to balance the current load rather than the historical one. Hence, the sliding window model is an active research field [3, 33, 36, 40, 41].

The desired measurement types differ from one application to the other. For example, a load balancer may be interested in the heavy hitter flows [1], which are responsible for a large portion of the traffic. Additionally, anomaly detection systems often monitor the number of distinct elements [24] and entropy [37] or use Bloom filters [5]. Yet, existing algorithms usually provide just a single utility at a time, e.g., approximate set membership (Bloom filters) [6], per-flow counting [12, 17], count distinct $[15,19,20]$ and entropy [37]. Therefore, as networks complexity grows, multiple measurement types may be required. However, employing multiple stand-alone solutions incurs the additive combined cost of each of them, which is inefficient in both memory and computation.

In this work, we suggest Sliding Window Approximate Measurement Protocol (SWAMP), an algorithm that bundles together four commonly used measurement types. Specifically, it approximates set membership, per flow counting, distinct elements and entropy in the sliding window model. As illustrated in Figure 1, SWAMP stores flows fingerprints ${ }^{1}$ in a cyclic buffer while their frequencies are maintained in a compact fingerprint hash table named TinyTable [17]. On each packet arrival, its corresponding fingerprint replaces the oldest one in the buffer. We then update the table, decrementing the departing fingerprint's frequency and incrementing that of the arriving one. An additional counter $Z$ maintains the number of distinct fingerprints in the window and is updated every time a fingerprint's frequency is reduced to 0 or increased to 1 . Intuitively, the number of distinct fingerprints provides a good estimation of the number of distinct elements. Additionally, the scalar $\widehat{H}$ (not illustrated) maintains the fingerprints distribution entropy and approximates the real entropy.

\footnotetext{
${ }^{1} \mathrm{~A}$ fingerprint is a short random string obtained by hashing an ID.
} 


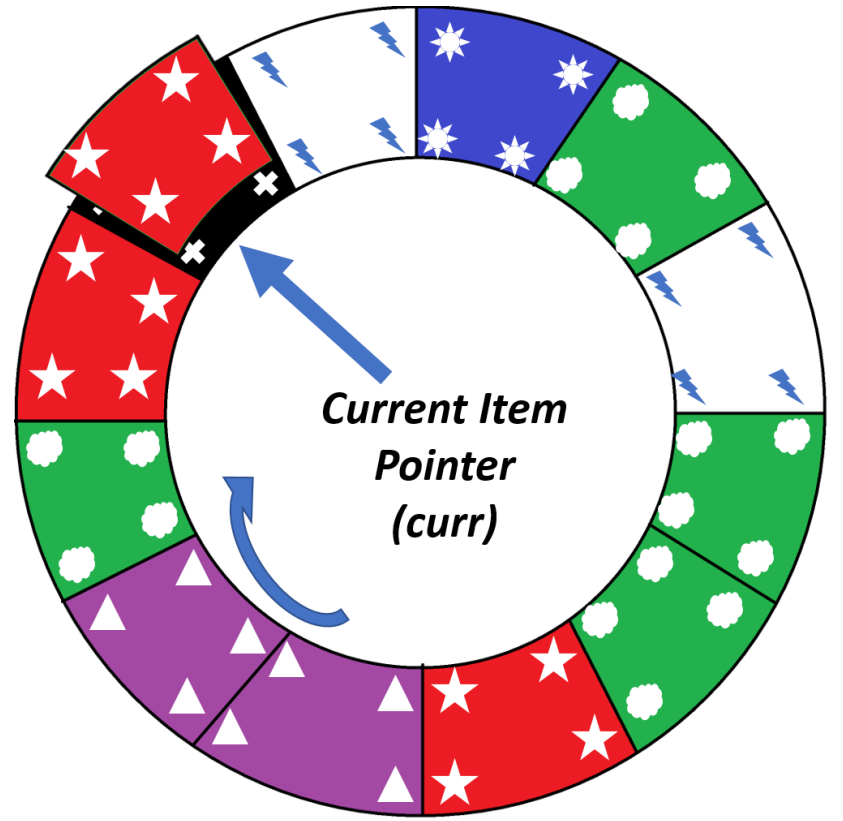

Cyclic Fingerprint Buffer (CFB)
Stream items: $x_{n-W} \quad x_{n-W+1} \ldots x_{n-1}$

$\begin{array}{lll}\text { Fingerprints: } & \boldsymbol{x} * \boldsymbol{t} \\ \text { New item: } & x_{n} \quad h\left(x_{n}\right)=\star \lambda\end{array}$

Distinct (Z): $6(-1)$

\begin{tabular}{|c|c|c|c|}
\hline \multicolumn{3}{|c|}{ Fingerprint } & Frequency \\
\hline$\star$ & $\star x$ & $\star$ & $2(+1)$ \\
\hline $\boldsymbol{x}$ & $\boldsymbol{x}$ & $x$ & $1(-1)$ \\
\hline$\Delta$ & $\Lambda$ & $\Delta$ & 2 \\
\hline 1 & 1 & 1 & 2 \\
\hline$\infty$ & 0 & 0 & 4 \\
\hline 来 & 米 & 米 & 1 \\
\hline
\end{tabular}

Figure 1: An overview of SWAMP: Fingerprints are stored in a cyclic fingerprint buffer (CFB), and their frequencies are maintained by TinyTable. Upon item $x_{n}$ 's arrival, we update CFB and the table by removing the oldest item's $\left(x_{n-W}\right)$ fingerprint (in black) and adding that of $x_{n}$ (in red). We also maintain an estimate for the number of distinct fingerprints $(\mathrm{Z})$. Since the black fingerprints count is now zero, we decrement $\mathrm{Z}$.

\subsection{Contribution}

We present $S W A M P$, a sliding window algorithm for approximate set membership (Bloom filters), per-flow counting, distinct elements and entropy measurements. We prove that SWAMP operates in constant time and provides accuracy guarantees for each of the supported problems. Despite its versatility, SWAMP improves the state of the art for each.

For approximate set membership, SWAMP is memory succinct when the false positive rate is constant and requires up to $40 \%$ less space than [33]. SWAMP is also succinct for per-flow counting and is more accurate than [3] on real packet traces. When compared with $1+\varepsilon$ count distinct approximation algorithms [9, 22], SWAMP asymptotically improves the query time from $O\left(\varepsilon^{-2}\right)$ to a constant. It is also up to x1000 times more accurate on real packet traces. For entropy, SWAMP asymptotically improves the runtime to a constant and provides accurate estimations in practice.

\begin{tabular}{|c|c|c|c|}
\hline Algorithm & Space & Time & Counts \\
\hline \hline SWAMP & $(1+o(1)) \cdot W \log _{2} W$ & $O(1)$ & $\boldsymbol{\nearrow}$ \\
\hline SWBF [33] & $(2+o(1)) \cdot W \log _{2} W$ & $O(1)$ & $\boldsymbol{x}$ \\
\hline TBF $[41]$ & $O\left(W \log _{2} W \log _{2} \epsilon^{-1}\right)$ & $O\left(\log _{2} \epsilon^{-1}\right)$ & $\boldsymbol{x}$ \\
\hline
\end{tabular}

Table 1: Comparison of sliding window set membership algorithms for $\epsilon=W^{-o(1)}$.

the notations we use can be found in Table 3 .

\subsection{Paper organization}

Related work on the problems covered by this work is found in Section 2. Section 3 provides formal definitions and introduces SWAMP. Section 4 describes an empirical evaluation of SWAMP and previously suggested algorithms. 


\begin{tabular}{|c|c|c|c|}
\hline Problem & Estimator & Guarantee & Reference \\
\hline \multirow{2}{*}{$(W, \epsilon)$-Approximate Set Membership } & \multirow{2}{*}{$\operatorname{IsMeMBer}()$} & $\operatorname{Pr}\left(\right.$ true $\left.\mid x \in S^{W}\right)=1$ & \multirow{2}{*}{ Corollary 5.4} \\
\hline & & $\operatorname{Pr}\left(\right.$ true $\left.\mid x \notin S^{W}\right) \leq \epsilon$ & \\
\hline \multirow[b]{2}{*}{$(W, \epsilon)$-Approximate Set Multiplicity } & \multirow{2}{*}{ FREQUENCY $\left(\widehat{f}_{x}\right)$} & $\operatorname{Pr}\left(f_{x} \leq \widehat{f}_{x}\right)=1$ & \multirow[b]{2}{*}{ Theorem 5.2} \\
\hline & & $\operatorname{Pr}\left(f_{x} \neq \widehat{f_{x}}\right) \leq \epsilon$ & \\
\hline \multirow{3}{*}{$(W, \epsilon, \delta)$-Approximate Count Distinct } & \multirow{2}{*}{ DistinctLB $(Z)$} & $\operatorname{Pr}(D \geq Z)=1$ & \multirow[b]{2}{*}{ Theorem 5.9} \\
\hline & & $\operatorname{Pr}\left(D-Z \geq \frac{1}{2} \varepsilon D \cdot \log \left(\frac{2}{\delta}\right)\right) \leq \delta$ & \\
\hline & DistinctMLE $(\widehat{D})$ & $\operatorname{Pr}\left(|D-\widehat{D}| \geq \frac{1}{2} \varepsilon D \cdot \log \left(\frac{2}{\delta}\right)\right) \leq \delta$ & Theorem 5.15 \\
\hline \multirow{2}{*}{$(W, \epsilon, \delta)$-Entropy Estimation } & \multirow{2}{*}{ ENTROPY $(\widehat{H})$} & $\operatorname{Pr}(H \geq \widehat{H})=1$ & \multirow[b]{2}{*}{ Theorem 5.17} \\
\hline & & $\operatorname{Pr}\left(H-\widehat{H} \geq \epsilon \delta^{-1}\right) \leq \delta$ & \\
\hline
\end{tabular}

Table 2: Summary of SWAMP's accuracy guarantees.

Section 5 includes a formal analysis of SWAMP which is briefly summarized in Table 2. Finally, we conclude with a short discussion in Section 6 .

\section{Related work}

\subsection{Set Membership and Counting}

A Bloom filter [6] is an efficient data structure that encodes an approximate set. Given an item, a Bloom filter can be queried if that item is a part of the set. An answer of 'no' is always correct, while an answer of 'yes' may be false with a certain probability. This case is called False Positive.

Plain Bloom filters do not support removals or counting and thus many algorithms fill this gap. For example, some alternatives support removals $[7,17,18,30,32,39]$ and others support multiplicity queries [12, 17]. Additionally, some works use aging [40] and others compute the approximate set with regard to a sliding windows $[41,33]$.

SWBF [33] uses a Cuckoo hash table to build a sliding Bloom filter, which is more space efficient than previously suggested Timing Bloom filters (TBF) [41].

The Cuckoo table is allocated with $2 W$ entries such that each entry stores a fingerprint and a time stamp. Cuckoo tables require that $W$ entries remain empty to avoid circles and this is done implicitly by treating cells containing outdated items as 'empty'. Finally, a cleanup process is used to remove outdated items and allow timestamps to be wrapped around. A comparison of SWAMP, TBF and SWBF appears in Table 1.

\subsection{Count Distinct}

The number of distinct elements provides a useful indicator for anomaly detection algorithms. Accurate count distinct is impractical due to the massive scale of the data [21] and thus most approaches resort to approximate solutions $[2,11,15]$.

Approximate algorithms typically use a hash function $H: \mathbb{D D} \rightarrow\{0,1\}^{\infty}$ that maps ids to infinite bit strings. In practice, finite bit strings are used and 32 bit integers suffice to reach estimations of over $10^{9}$ [21]. These algorithms look for certain observables in the hashes. For example, some algorithms $[2,25]$ treat the minimal observed hash value as a real number in $[0,1]$ and exploit the fact that $\mathbb{E}(\min (H(\mathcal{M})))=\frac{1}{D+1}$, where $D$ is the real number of distinct items in the multi-set $\mathcal{M}$. Alternatively, one can seek patterns of the form $0^{\beta-1} 1[15,21]$ and exploit the fact that such a pattern is encountered on average once per every $2^{\beta}$ unique elements.

Monitoring observables reduces the required amount of space as we only need to maintain a single one. In practice, the variance of such methods is large and hence multiple observables are maintained. 
In principle, one could repeat the process and perform $m$ independent experiments but this has significant computational overheads. Instead, stochastic averaging [20] is used to mimic the effects of multiple experiments with a single hash calculation. At any case, using $m$ repetitions reduces the standard deviation by a factor of $\frac{1}{\sqrt{m}}$.

The state of the art count distinct algorithm is HyperLogLog (HLL) [21], which is used in multiple Google projects [27]. HLL requires $m$ bytes and its standard deviation is $\sigma \approx \frac{1.04}{\sqrt{m}}$. SWHLL extends HLL to sliding windows $[9,22]$, and was used to detect attacks such as port scans [10]. SWAMP's space requirement is proportional to $W$ and thus, it is only comparable in space to HLL when $\varepsilon^{-2}=O(W)$. However, when multiple functionalities are required the residual space overhead of SWAMP is only $\log (W)$ bits, which is considerably less than any standalone alternative.

\subsection{Entropy Detection}

Entropy is commonly used as a signal for anomaly detection [37]. Intuitively, it can be viewed as a summary of the entire traffic histogram. The benefit of entropy based approaches is that they require no exact understanding of the attack's mechanism. Instead, such a solution assumes that a sharp change in the entropy is caused by anomalies.

An $\epsilon, \delta$ approximation of the entropy of a stream can be calculated in $O\left(\varepsilon^{-2} \log \delta^{-1}\right)$ space [26], an algorithm that was also extended to sliding window using priority sampling [14]. That sliding window algorithm is improved by [8] whose algorithm requires $O\left(\varepsilon^{-2} \log \delta^{-1} \log (N)\right)$ memory.

\subsection{Preliminaries - Compact Set Multiplicity}

Our work requires a compact set multiplicity structure that support both set membership and multiplicity queries. TinyTable [17] and CQF [38] fit the description while other structures [7, 30] are naturally expendable for multiplicity queries with at the expense of additional space. We choose TinyTable [17] as its code is publicly available as open source.

TinyTable encodes $W$ fingerprints of size $L$ using $(1+\alpha) W\left(L-\log _{2}(W)+3\right)+o(W)$ bits, where $\alpha$ is a small constant that affects update speed; when $\alpha$ grows, TinyTable becomes faster but also consumes more space.

\section{SWAMP Algorithm}

\subsection{Model}

We consider a stream $(\mathbb{S})$ of IDs where at each step an ID is added to $\mathbb{S}$. The last $W$ elements in $\mathbb{S}$ are denoted $\mathbb{S}^{W}$. Given an ID $y$, the notation $f_{y}^{W}$ represents the frequency of $y$ in $\mathbb{S}^{W}$. Similarly, $\widehat{f_{y}^{W}}$ is an approximation of $f_{y}^{W}$. For ease of reference, notations are summarized in Table 3.

\subsection{Problems definitions}

We start by formally defining the approximate set membership problem.

Definition 1. We say that an algorithm solves the $(W, \epsilon)$-Approximate SeT Membership problem if given an ID $y$, it returns true if $y \in \mathbb{S}^{W}$ and if $y \notin \mathbb{S}^{W}$, it returns false with probability of at least $1-\varepsilon$.

The above problem is solved by SWBF [33] and by Timing Bloom filter (TBF) [41]. In practice, SWAMP solves the stronger $(W, \epsilon)$-Approximate SeT Multiplicity problem, as defined below:

Definition 2. We say that an algorithm solves the $(W, \epsilon)$-ApProximate SET Multiplicity problem if given an ID $y$, it returns an estimation $\widehat{f_{y}^{W}}$ s.t. $\widehat{f_{y}^{W}} \geq f_{y}^{W}$ and with probability of at least $1-\varepsilon: f_{y}{ }^{W}=\widehat{f_{y}{ }^{W}}$.

Intuitively, the $(W, \epsilon)$-Approximate Set Multiplicity problem guarantees that we always get an over approximation of the frequency and that with probability of at least $1-\varepsilon$ we get the exact window frequency. A simple observation shows that any algorithm that solves the $(W, \epsilon)$-Approximate Set Multiplicity problem also solves the $(W, \epsilon)$-Approximate Set Membership problem. Specifically, if $y \in \mathbb{S}^{W}$, then $\widehat{f_{y}^{W}} \geq f_{y}^{W}$ implies 


\begin{tabular}{|c|l|}
\hline Symbol & Meaning \\
\hline$W$ & Sliding window size \\
\hline $\mathbb{S}$ & Stream. \\
\hline $\mathbb{S}^{W}$ & Last $W$ elements in the stream. \\
\hline$\varepsilon$ & Accuracy parameter for sets membership. \\
\hline$\varepsilon_{D}$ & Accuracy parameter for count distinct. \\
\hline$\varepsilon_{H}$ & Accuracy parameter for entropy estimation. \\
\hline$\delta$ & Confidence for count distinct. \\
\hline$L$ & Fingerprint length in bits, $L \triangleq \log _{2}\left(W \varepsilon^{-1}\right)$. \\
\hline$f_{y}^{W}$ & Frequency of ID $y$ in $\mathbb{S}^{W}$. \\
\hline$f_{y}^{W}$ & An approximation of $f_{y}^{W}$. \\
\hline$D$ & Number of distinct elements in $\mathbb{S}^{W}$. \\
\hline$Z$ & Number of distinct fingerprints. \\
\hline$\widehat{D}$ & Estimation provided by DISTINCTMLE function. \\
\hline$\alpha$ & TinyTable's parameter $(\alpha=1.2)$. \\
\hline$h$ & A pairwise independent hash function. \\
\hline$F$ & A set of fingerprints stored in $C F B$. \\
\hline
\end{tabular}

Table 3: List of symbols

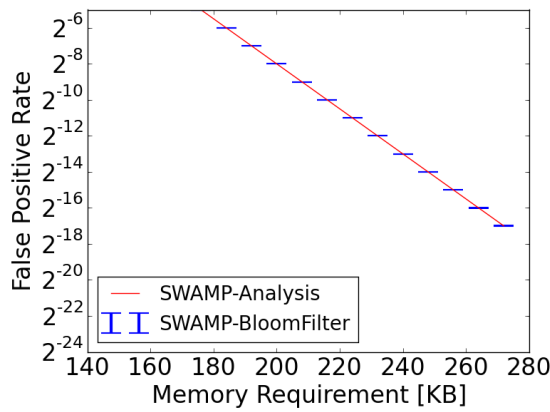

(a) Sliding Bloom filter

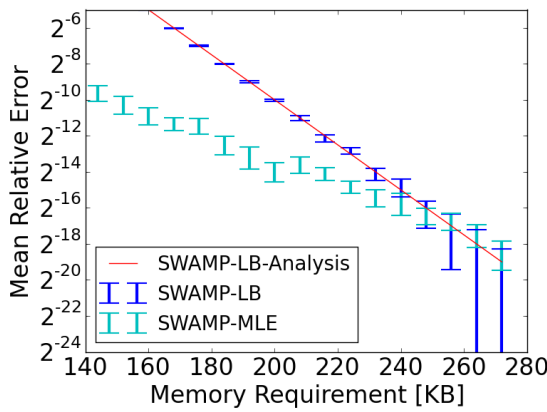

(b) Count Distinct

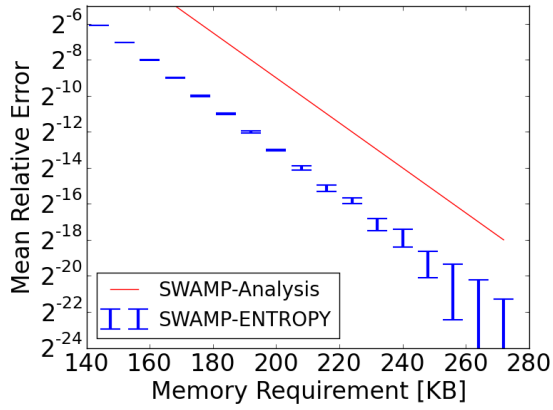

(c) Entropy

Figure 2: Empirical error and theoretical guarantee for multiple functionalities (random inputs).

that $\widehat{f_{y}^{W}} \geq 1$ and we can return true. On the other hand, if $y \notin \mathbb{S}^{W}$, then $f_{y}{ }^{W}=0$ and with probability of at least $1-\varepsilon$, we get: $f_{y}{ }^{W}=0=\widehat{f_{y}{ }^{W}}$. Thus, the isMember estimator simply returns true if $\widehat{f_{y}{ }^{W}}>0$ and false otherwise. We later show that this estimator solves the $(W, \epsilon)$-Approximate Set Membership problem.

The goal of the $(W, \epsilon, \delta)$-Approximate Count Distinct problem is to maintain an estimation of the number of distinct elements in $\mathbb{S}^{W}$. We denote their number by $D$.

Definition 3. We say that an algorithm solves the $(W, \epsilon, \delta)$-Approximate Count Distinct problem if it returns an estimation $\widehat{D}$ such that $D \geq \widehat{D}$ and with probability $1-\delta: \widehat{D} \geq(1-\varepsilon) D$.

Intuitively, an algorithm that solves the $(W, \epsilon, \delta)$-Approximate Count Distinct problem is able to conservatively estimate the number of distinct elements in the window and with probability of $1-\delta$, this estimate is close to the real number of distinct elements. 


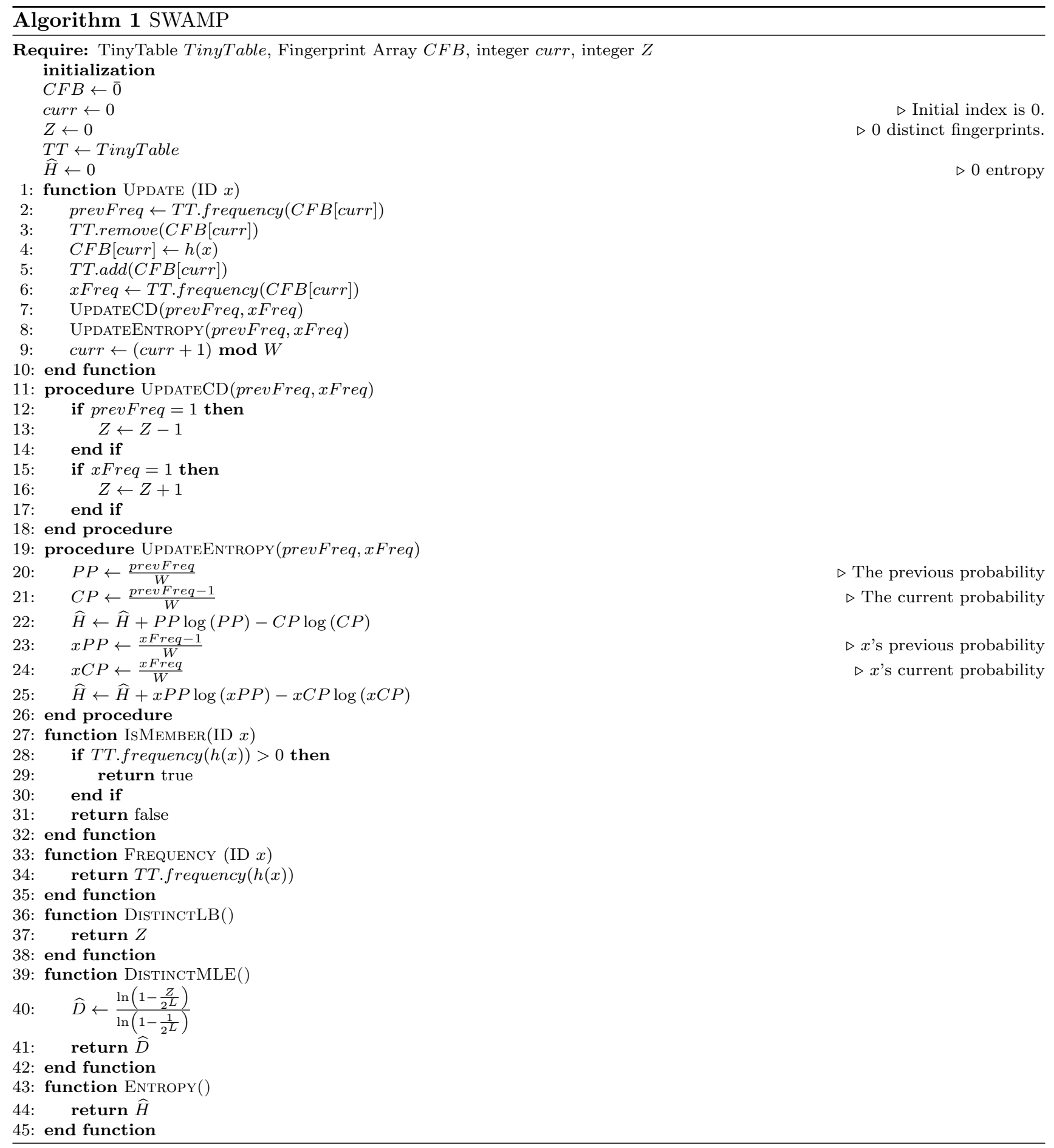

The entropy of a window is defined as:

$$
H \triangleq-\sum_{i=1}^{D} \frac{f_{i}}{W} \log \left(\frac{f_{i}}{W}\right),
$$

where $D$ is the number of distinct elements in the window, $W$ is the total number of packets, and $f_{i}$ is the frequency of flow $i$. We define the window entropy estimation problem as: 


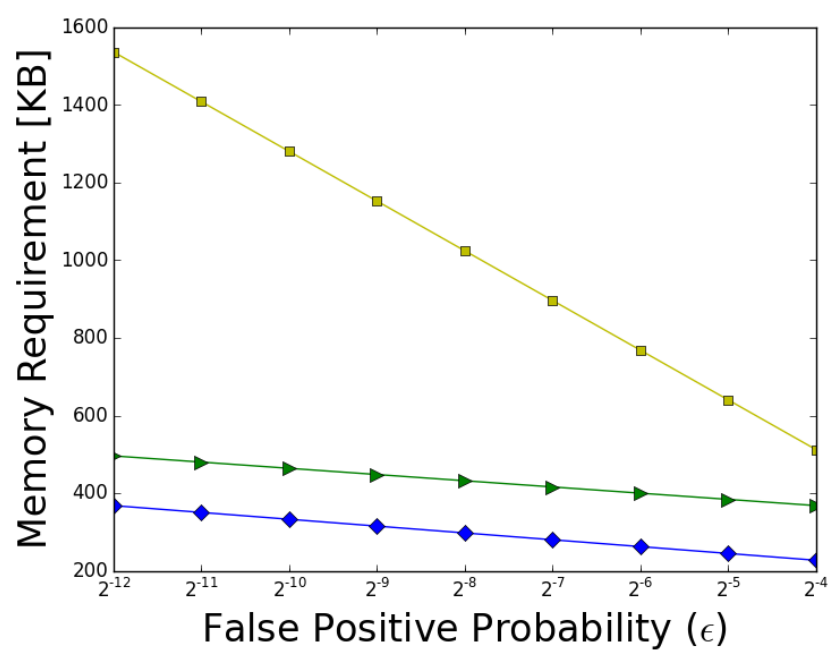

(a) Window size is $2^{16}$ and varying $\varepsilon$.

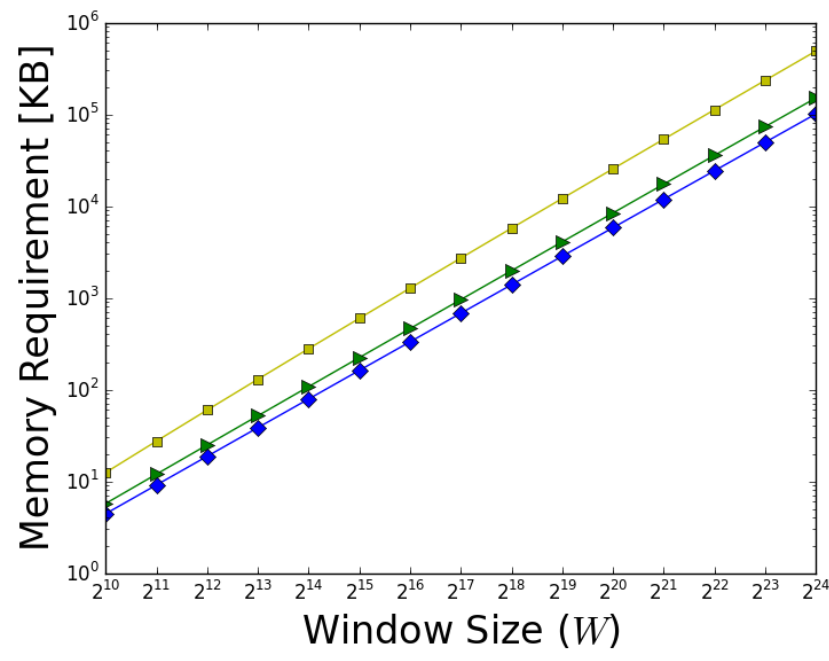

(b) $\varepsilon=2^{-10}$ and varying window sizes.

$\bullet$ SWAMP $\longmapsto$ SWBF $\square$ SBF

Figure 3: Memory consumption of sliding Bloom filters as a function of $W$ and $\varepsilon$.

Definition 4. An algorithm solves the $(W, \epsilon, \delta)$-ENTROPY Estimation problem, if it provides an estimator $\widehat{H}$ so that $H \geq \widehat{H}$ and $\operatorname{Pr}(H-\widehat{H} \geq \epsilon) \leq \delta$.

\subsection{SWAMP algorithm}

We now present Sliding Window Approximate Measurement Protocol (SWAMP). SWAMP uses a single hash function $(h)$, which given an ID $(y)$, generates $L \triangleq\left\lceil\log _{2}\left(W \epsilon^{-1}\right)\right\rceil$ random bits $h(y)$ that are called its fingerprint. We note that $h$ only needs to be pairwise-independent and can thus be efficiently implemented using only $O(\log W)$ space. Fingerprints are then stored in a cyclic fingerprint buffer of length $W$ that is denoted $C F B$. The variable curr always points to the oldest entry in the buffer. Fingerprints are also stored in TinyTable [17] that provides compact encoding and multiplicity information.

The update operation replaces the oldest fingerprint in the window with that of the newly arriving item. To do so, it updates both the cyclic fingerprint buffer $(C F B)$ and TinyTable. In CFB, the fingerprint at location curr is replaced with the newly arriving fingerprint. In TinyTable, we remove one occurrence of the oldest fingerprint and add the newly arriving fingerprint. The update method also updates the variable $Z$, which measures the number of distinct fingerprints in the window. $Z$ is incremented every time that a new unique fingerprint is added to TinyTable, i.e., FREQUENCY $(y)$ changes from 0 to 1, where the method FREQUENCY $(y)$ receives a fingerprint and returns its frequency as provided by TinyTable. Similarly, denote $x$ the item whose fingerprint is removed; if $F R E Q U E N C Y(x)$ changes to 0, we decrement $Z$.

SWAMP has two methods to estimate the number of distinct flows. DistinctLB simply returns $Z$, which yields a conservative estimator of $D$ while DistinctMLE is an approximation of its Maximum Likelihood Estimator. Clearly, DistinctMLE is more accurate than DistinctLB, but its estimation error is two sided. A pseudo code is provided in Algorithm 1 and an illustration is given by Figure 1. 


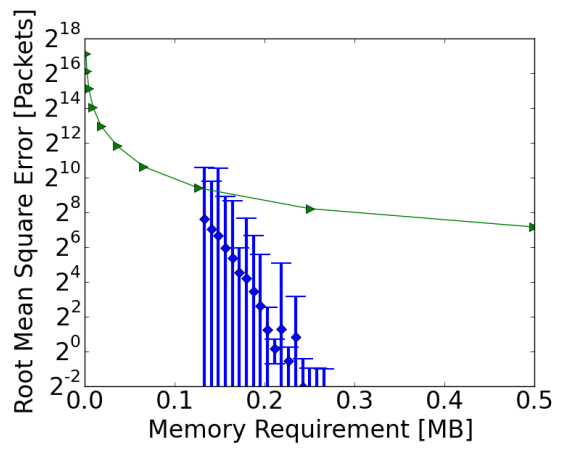

(a) Chicago 16 Dataset

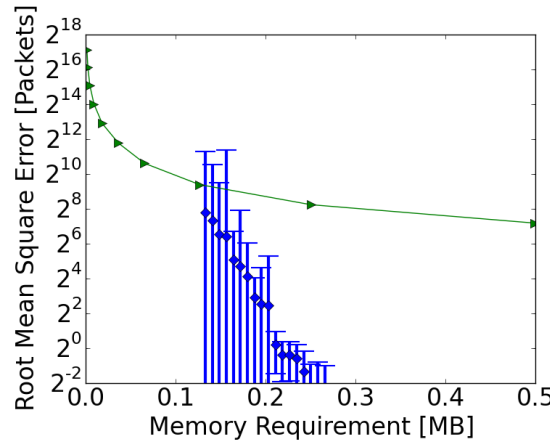

(d) Chicago 15 Dataset

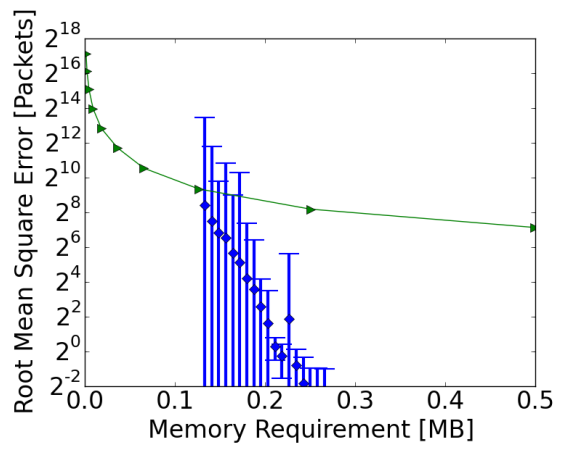

(b) San Jose 14 Dataset

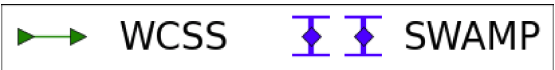

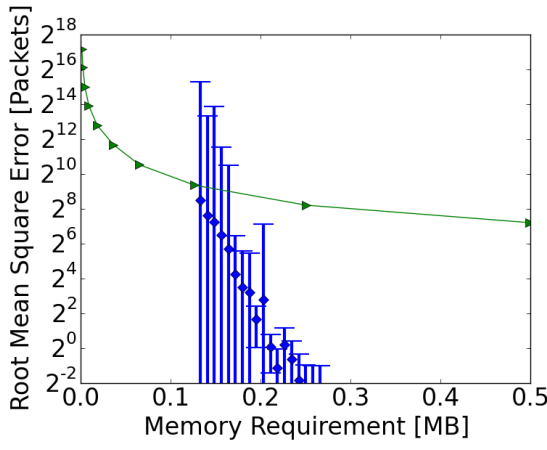

(e) San Jose 13 Dataset

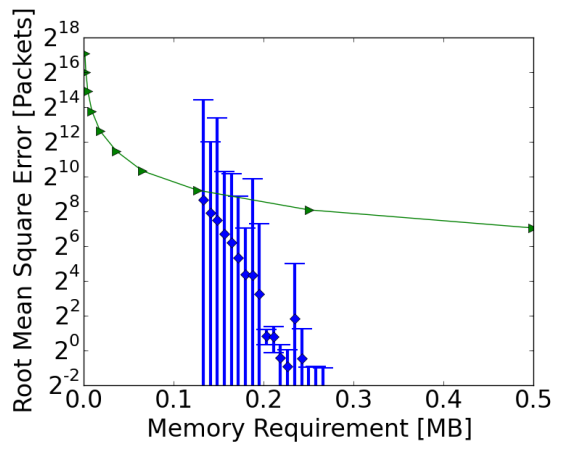

(c) DC 1 Dataset

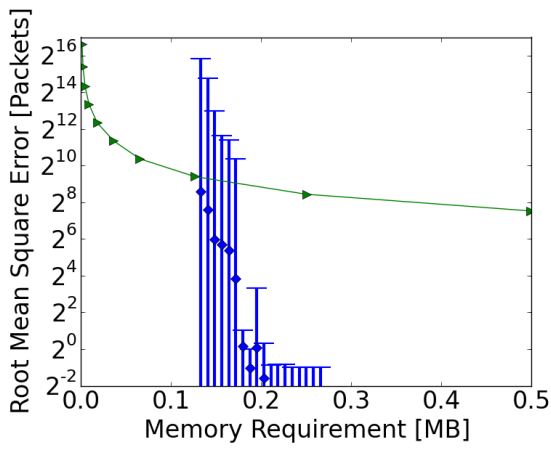

(f) DC 2 Dataset

Figure 4: Space/accuracy trade-off in estimating per flow packet counts (window size: $W=2^{16}$ ).

\section{Empirical Evaluation}

\subsection{Overview}

We evaluate SWAMP's various functionalities, each against its known solutions. We start with the $(W, \epsilon)$ Approximate Set Membership problem where we compare SWAMP to SWBF [33] and Timing Bloom filter (TBF) [41], which only solve $(W, \epsilon)$-Approximate Set Membership.

For counting, we compare SWAMP to Window Compact Space Saving (WCSS) [3] that solves heavy hitters identification on a sliding window. WCSS provides a different accuracy guarantee and therefore we evaluate their empirical accuracy when both algorithms are given the same amount of space.

For the distinct elements problem, we compare SWAMP against Sliding Hyper Log Log [9, 22], denoted SWHLL, who proposed running HyperLogLog and LogLog on a sliding window. In our settings, small range correction is active and thus HyperLogLog and LogLog devolve into the same algorithm. Additionally, since we already know that small range correction is active, we can allocate only a single bit (rather than 5) to each counter. This option, denoted SWLC, slightly improves the space/accuracy ratio.

In all measurements, we use a window size of $W=2^{16}$ unless specified otherwise. In addition, the underlying TinyTable uses $\alpha=0.2$ as recommended by its authors [17].

Our evaluation includes six Internet packet traces consisting of backbone routers and data center traffic. The backbone traces contain a mix of 1 billion UDP, TCP and ICMP packets collected from two major routers in Chicago [29] and San Jose [28] during the years 2013-2016. The dataset Chicago 16 refers to data collected from the Chicago router in 2016, San Jose 14 to data collected from the San Jose router in 2014, etc. The datacenter packet traces are taken from two university datacenters that consist of up to 1,000 servers [4]. These traces are 


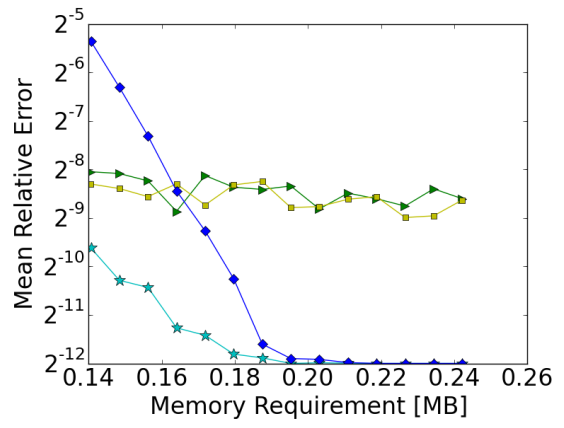

(a) Chicago 16 Dataset

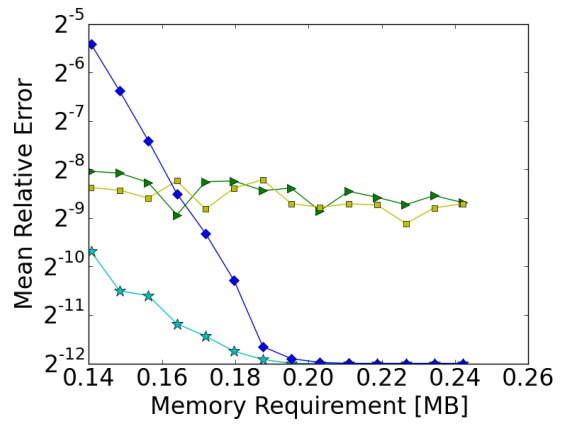

(b) San Jose 14 Dataset

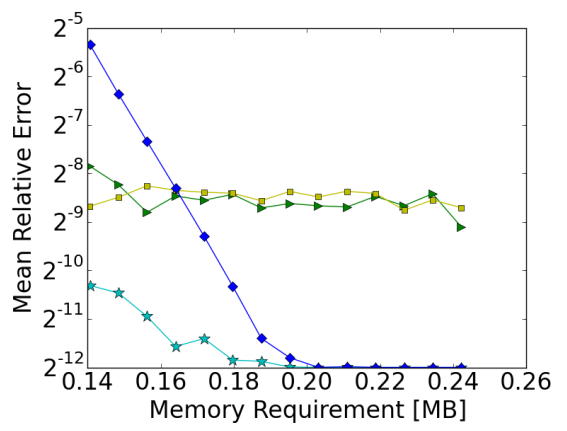

(c) DC 1 Dataset

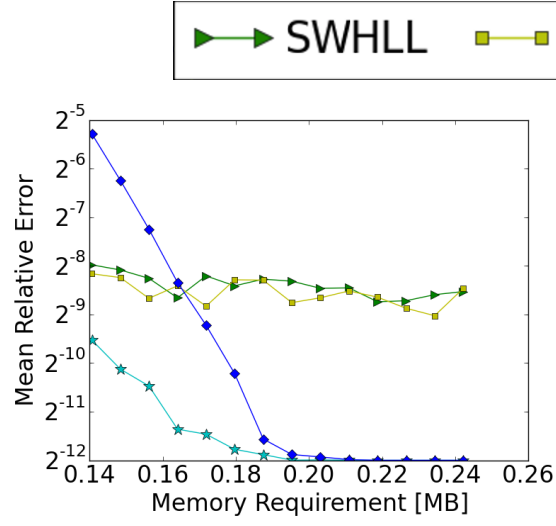

(d) Chicago 15 Dataset

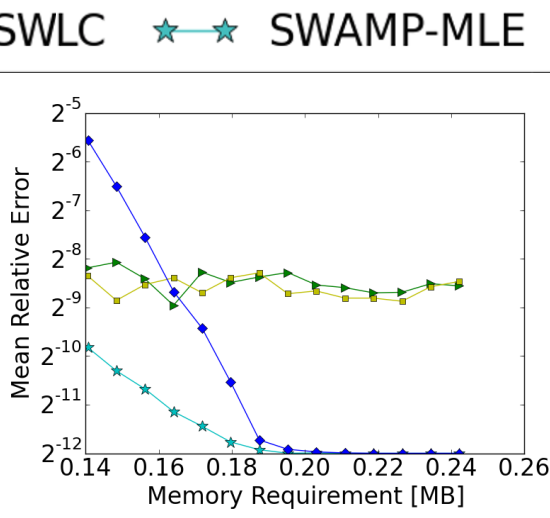

(e) San Jose 13 Dataset

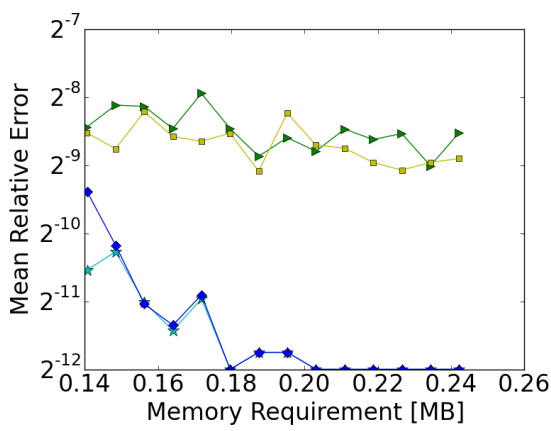

(f) DC 2 Dataset

Figure 5: Accuracy of SWAMP's count distinct functionality compared to alternatives.

denoted DC1 and DC2.

\subsection{Evaluation of analytical guarantees}

Figure 2 evaluates the accuracy of our analysis from Section 5 on random inputs. As can be observed, the analysis of sliding Bloom filter (Figure 2(a)) and count distinct (Figure 2(b)) is accurate. For entropy (Figure 2(c)) the accuracy is better than anticipated indicating that our analysis here is just an upper bound, but the trend line is nearly identical.

\subsection{Set membership on sliding windows}

We now compare SWAMP to TBF [41] and SWBF [33]. Our evaluation focuses on two aspects, fixing $\varepsilon$ and changing the window size (Figure 3(b)) as well as fixing the window size and changing $\varepsilon$ (Figure 3(a)).

As can be observed, SWAMP is considerably more space efficient than the alternatives in both cases for a wide range of window sizes and for a wide range of error probabilities. In the tested range, it is $25-40 \%$ smaller than the best alternative.

\subsection{Per-flow counting on sliding windows}

Next, we evaluate SWAMP for its per-flow counting functionality. We compare SWAMP to WCSS [3] that solves heavy hitters on a sliding window. Our evaluation uses the On Arrival model, which was used to evaluate WCSS. In that model, we perform a query for each incoming packet. Then, we calculate the Root Mean Square Error. We repeated each experiment 25 times with different seeds and computed 95\% confidence intervals for SWAMP. Note that WCSS is a deterministic algorithm and as such was run only once. 


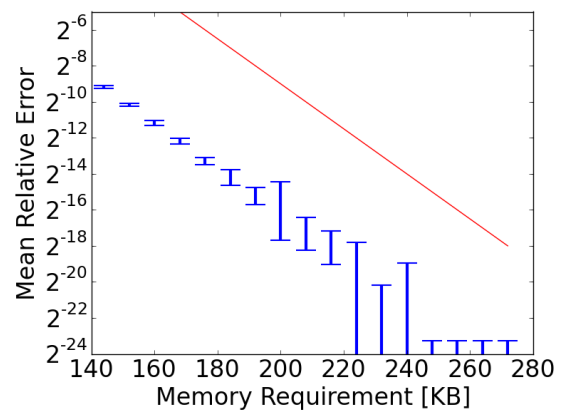

(a) Chicago 16 Dataset

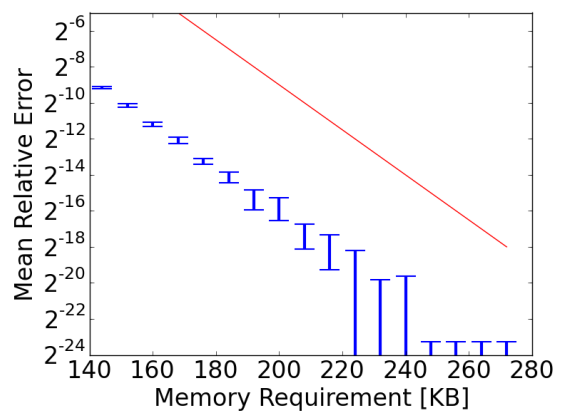

(b) San Jose 14 Dataset

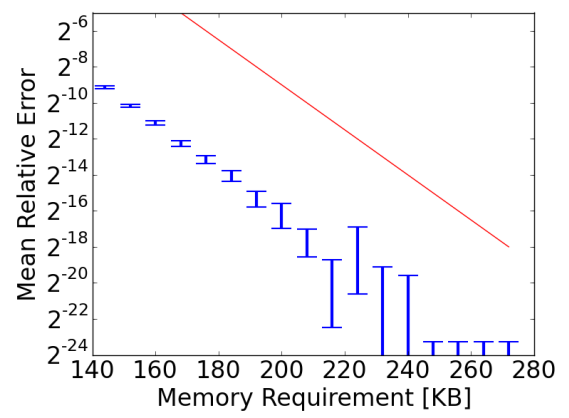

(c) DC 1 Dataset

\section{- SWAMP-Analysis II SWAMP-ENTROPY}

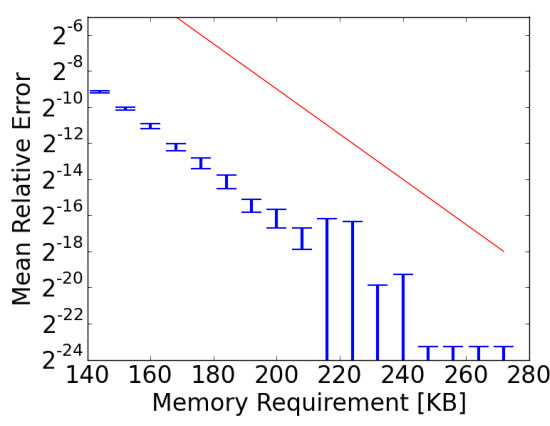

(d) Chicago 15 Dataset

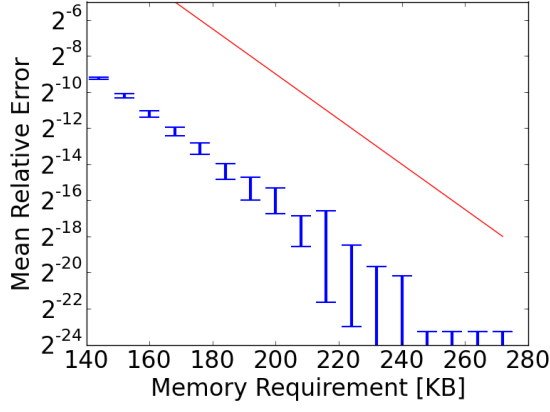

(e) San Jose 13 Dataset

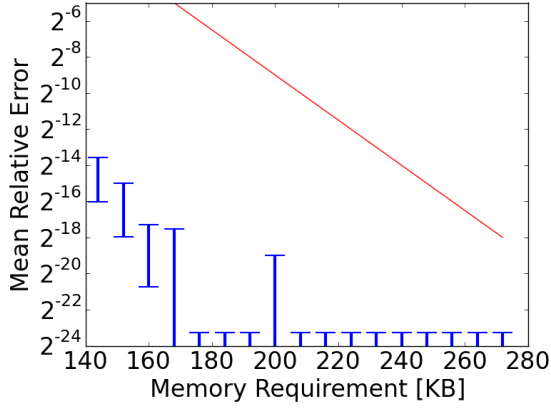

(f) DC 2 Dataset

Figure 6: Accuracy of SWAMP's entropy functionality compared to the theoretical guarantee.

The results appear in Figure 4. Note that the space consumption of WCSS is proportional to $\varepsilon$ and that of SWAMP to $W$. Thus, SWAMP cannot be run for the entire range. Yet, when it is feasible, SWAMP's error is lower on average than that of WCSS. Additionally, in many of the configurations we are able to show statistical significance to this improvement. Note that SWAMP becomes accurate with high probability using about 300KB of memory while WCSS requires about 8.3MB to provide the same accuracy. That is, an improvement of x27.

\subsection{Count distinct on sliding windows}

Next, we evaluate the count distinct functionality in terms of accuracy vs. space on the different datasets. We performed 25 runs and summarized the averaged the results. We evaluate two functionalities: SWAMP-LB and SWAMP-MLE. SWAMP-LB corresponds to the function DistinctLB in Algorithm 1 and provides one sided estimation while SWAMP-MLE corresponds to the function DistinctMLE in Algorithm 1 and provides an unbiased estimator.

Figure 5 shows the results of this evaluation. As can be observed, SWAMP-MLE is up to x1000 more accurate than alternatives. Additionally, SWAMP-LB also out performs the alternatives for parts of the range. Note that SWAMP-LB is the only one sided estimator in this evaluation.

\subsection{Entropy estimation on sliding window}

Figure 6 shows results for Entropy estimation. As shown, SWAMP provides a very accurate entropy estimation in its entire operational range. Moreover, our analysis in Section 5.3 is conservative and SWAMP is much more accurate in practice. 


\section{Analysis of SWAMP}

This section is dedicated for the analysis of SWAMP. Our analysis is partitioned into three subsections. Section 5.1 shows that SWAMP solves the $(W, \epsilon)$-Approximate Set Membership and the $(W, \epsilon)$-Approximate SET Multiplicity problems and explains the conditions where it is succinct. It also shows that SWAMP operates in constant runtime. Next, Section 5.2 shows that SWAMP solves the $(W, \epsilon, \delta)$-Approximate Count Distinct problem using DistinctLB and that DistinctMLE approximates its Maximum Likelihood Estimator. Finally, Section 5.3 shows that SWAMP solves $(W, \epsilon, \delta)$-Entropy Estimation.

\subsection{Analysis of per flow counting}

We start by showing that SWAMP's runtime is constant.

Theorem 5.1. SWAMP's runtime is O(1) with high probability.

Proof. Update - Updating the cyclic buffer requires two TinyTable operations - add and remove - both performed in constant time (with high probability) for any constant $\alpha$. The manipulations to $\widehat{H}$ and $Z$ are also done in constant time.

ISMEMBER - Is satisfied by TinyTable in constant time.

FREQUENCY - Is satisfied by TinyTable in constant time.

DisTINCTLB - Is satisfied by returning an integer.

DisTINCTMLE - Is satisfied with a simple calculation.

ENTROPY - Is satisfied by returning a floating point.

Next, we prove that SWAMP solves the $(W, \epsilon)$-Approximate Set Multiplicity problem with regard to the function FREQUENCY (in Algorithm 1).

Theorem 5.2. SWAMP solves the $(W, \epsilon)$-Approximate Set Multiplicity problem. That is, given an ID $y$, FREQUENCY $(y)$ provides an estimation $\widehat{f_{y}^{W}}$ such that: $\widehat{f_{y}^{W}} \geq f_{y}^{W}$ and $\operatorname{Pr}\left[f_{y}^{W}=\widehat{f_{y}^{W}}\right] \geq 1-\varepsilon$.

Proof. SWAMP's $C F B$ variable stores $W$ different fingerprints, each of length $L=\log _{2}\left(W \epsilon^{-1}\right)$. For a fingerprint of size $L$, the probability that two fingerprints collide is $2^{-L}$. There are $W-1$ fingerprints that $h(y)$ may collide with, and $\widehat{f_{y}^{W}}>f_{y}^{W}$ only if it collides with one of the fingerprints in $C F B$. Next, we use the Union Bound to get:

$$
\operatorname{Pr}\left[f_{y}^{W} \neq \widehat{f_{y}^{W}}\right] \leq W \cdot 2^{-\log _{2}\left(W \epsilon^{-1}\right)}=\varepsilon .
$$

Thus, given an item $y$, with probability $1-\varepsilon$, its fingerprint $(h(y))$ is unique and TinyTable accurately measures $f_{y}^{W}$. Any collision of $h(y)$ with other fingerprints only increases $\widehat{f_{y}^{W}}$, thus $\widehat{f_{y}^{W}} \geq f_{y}^{W}$ in all cases.

Theorem 5.2 shows that SWAMP solves the $(W, \epsilon)$-Approximate Set Multiplicity, which includes per flow counting and sliding Bloom filter functionalities.

Our next step is to analyze the space consumption of SWAMP. This enables us to show that SWAMP is memory optimal to the $(W, \epsilon)$-Approximate Set Multiplicity, or more precisely, that it is succinct (according to Definition 5).

Definition 5. Given an information theoretic lower bound $\mathcal{B}$, an algorithm is called succinct if it uses $\mathcal{B}(1+$ $o(1))$ bits.

We now analyze the space consumption of SWAMP.

Theorem 5.3. Given a window size $W$ and an accuracy parameter $\varepsilon$, the number of bits required by $S W A M P$ is: $W\left(\left\lceil\log _{2}\left(W \epsilon^{-1}\right)\right\rceil+(1+\alpha)\left(\log _{2} \epsilon^{-1}+3\right)\right)+o(W)$. 
Proof. Our cyclic buffer $(C F B)$ stores $W$ fingerprints, each of size $\left[\log _{2}\left(W \epsilon^{-1}\right)\right\rceil$, for an overall space of $W\left\lceil\log _{2}\left(W \epsilon^{-1}\right)\right\rceil$ bits. Additionally, TinyTable requires: $(1+\alpha) W\left(\log _{2}\left(W \epsilon^{-1}\right)-\log _{2}(W)+3\right)+o(W)$ $=(1+\alpha) W\left(\log _{2} \epsilon^{-1}+3\right)+o(W)$ bits.

Each one of the variables curr, $Z$ and $\widehat{H}$ require $\log (W)=o(W)$ and thus SWAMP's memory consumption is

$$
W\left(\left\lceil\log _{2}\left(W \epsilon^{-1}\right)\right\rceil+(1+\alpha)\left(\log _{2} \epsilon^{-1}+3\right)\right)+o(W) .
$$

The work of [36] implies a lower bound for the $(W, \epsilon)$-Approximate Set Membership problem of

$$
\mathcal{B} \triangleq W\left(\log _{2} \epsilon^{-1}+\max \left(\log _{2} \log _{2} \epsilon^{-1}, \log _{2}(W)\right)\right)
$$

bits. This lower bound also applies to our case, as SWAMP solves the $(W, \epsilon)$-Approximate SET MultiPlicity and the $(W, \epsilon)$-Approximate Set Membership by returning whether or not $\widehat{f_{y}{ }^{W}}>0$.

Corollary 5.4. SWAMP solves the $(W, \epsilon)$-Approximate Set Membership problem.

We now show that SWAMP is succinct for $\epsilon=W^{-o(1)}$.

Theorem 5.5. Let $\epsilon=W^{-o(1)}$. Then $S W A M P$ is succinct for the $(W, \epsilon)$-Approximate Set Membership and $(W, \epsilon)$-Approximate Set Multiplicity problems.

Proof. Theorem 5.3 shows that the required space is:

$$
\begin{aligned}
& S \leq W \log _{2} W+W(2+\alpha) \log _{2} \epsilon^{-1}+4 W+3 W \alpha+o(W)= \\
& W \log _{2} W \cdot\left(1+\frac{1}{\log _{2} W}\left[(2+\alpha) \log _{2} \epsilon^{-1}+4+3 \alpha+o(1)\right]\right)=W \log _{2} W \cdot\left(1+(2+\alpha) \frac{\log _{2} \epsilon^{-1}}{\log _{2} W}+o(1)\right) .
\end{aligned}
$$

We show that this implies succinctness according to Definition 5. Under our assumption that $\epsilon=W^{-o(1)}$, we get that: $\log _{2} \epsilon^{-1}=\log _{2}\left(W^{o(1)}\right)=o\left(\log _{2} W\right)$, hence SWAMP uses $W \log _{2} W(1+o(1))=\mathcal{B}(1+o(1))$ bits and is succinct.

\subsection{Analysis of count distinct functionality}

We now move to analyze SWAMP's count distinct functionality. Recall that SWAMP has two estimators; the one sided DistinctLB and the more accurate DistinctMLE. Also, recall that $Z$ monitors the number of distinct fingerprints and that $D$ is the real number of distinct flows (on the window).

\subsubsection{Analysis of DistinctLB}

We now present an analysis for DistinctLB, showing that it solves the $(W, \epsilon, \delta)$-Approximate Count Distinct problem.

Theorem 5.6. $Z \leq D$ and $\mathbb{E}(Z) \geq D \cdot\left(1-\frac{\varepsilon}{2}\right)$.

Proof. Clearly, $Z \leq D$ always, since any two identical IDs map to the same fingerprint. Next, for any $0 \leq i \leq$ $2^{L}-1$, denote by $Z_{i}$ the indicator random variable, indicating whether there is some item in the window whose fingerprint is $i$. Then $Z=\sum_{i=0}^{2^{L}-1} Z_{i}$, hence $\mathbb{E}(Z)=\sum_{i=0}^{2^{L}-1} \mathbb{E}\left(Z_{i}\right)$. However, for any $i$,

$$
\mathbb{E}\left(Z_{i}\right)=\operatorname{Pr}\left(Z_{i}=1\right)=1-\left(1-2^{-L}\right)^{D} .
$$

The probability that a fingerprint is exactly $i$ is $2^{-L}$, thus:

$$
\mathbb{E}(Z)=2^{L} \cdot\left(1-\left(1-2^{-L}\right)^{D}\right) .
$$

Since $0<2^{-L} \leq 1$, we have:

$\left(1-2^{-L}\right)^{D}<1-D \cdot 2^{-L}+\left(\begin{array}{l}D \\ 2\end{array}\right) \cdot 2^{-2 L}$ which, in turn, implies $\mathbb{E}(Z)>D-\left(\begin{array}{l}D \\ 2\end{array}\right) \cdot 2^{-L}$. Finally, we note that $2^{-L}=\frac{\varepsilon}{W}$ and that $D \leq W$. Hence, $\mathbb{E}(Z)>D-\frac{D(D-1) \varepsilon}{2 W}>D \cdot\left(1-\frac{\varepsilon}{2}\right)$. 
Our next step is a probabilistic bound on the error of $Z(X=D-Z)$. This is required for Theorem 5.9, which is the main result and shows that $Z$ provides an $\epsilon, \delta$ approximation for the distinct elements problem.

We model the problem as a balls and bins problem where $D$ balls are placed into $2^{L}=\frac{W}{\varepsilon}$ bins. The variable $X$ is the number of bins with at least 2 balls.

For any $0 \leq i \leq 2^{L}-1$, denote by $X_{i}$ the random variable denoting the number of balls in the $i$-th bin. Note that the variables $X_{i}$ are dependent of each other and are difficult to reason about directly. Luckily, $X$ is monotonically increasing and we can use a Poisson approximation. To do so, we denote by $Y_{i} \sim \operatorname{Poisson}\left(\frac{D}{2^{L}}\right)$ the corresponding independent Poisson variables and by $Y$ the Poisson approximation of $X$, that is $Y$ is the sum of $Y_{i}^{\prime} s$ with value 2 or more.

Our goal is to apply Lemma 5.7, which links the Poisson approximation to the exact case. In our case, it allows us to derive insight about $X$ by analyzing $Y$.

Lemma 5.7 (Corollary 5.11, page 103 of [35]). Let $\mathcal{E}$ be an event whose probability is either monotonically increasing or decreasing with the number of balls. If $\mathcal{E}$ has probability $p$ in the Poisson case then $\mathcal{E}$ has probability at most $2 p$ in the exact case. Here $\mathcal{E}$ is an event depending on $X_{0}, \ldots, X_{2^{L}-1}$ (in the exact case), and the probability of the event in the Poisson case is obtained by computing $\mathcal{E}$ using $Y_{0}, \ldots, Y_{2^{L}-1}$.

That is, we need to bound the probability $\operatorname{Pr}\left(X_{i} \geq 2\right)$ and to do so we define a random variable $Y$ and bound: $\operatorname{Pr}\left(Y_{i} \geq 2\right)$ which can be at $\tilde{\tilde{Y}}_{i} \sin 2 \cdot \operatorname{Pr}\left(X_{i} \geq 2\right)$.

We may now denote by $\tilde{Y}_{i}=Y_{i} \geq 2$ the indicator variables, indicating whether $Y_{i}$ has value at least 2 . By definition:

$Y=\sum_{i=0}^{2^{L}-1} \tilde{Y}_{i}$. It follows that:

$$
\mathbb{E}(Y)=\sum_{i=0}^{2^{L}-1} \mathbb{E}\left(\tilde{Y}_{i}\right)=2^{L} \cdot \operatorname{Pr}\left(Y_{i} \geq 2\right) .
$$

$Y_{i} \sim$ Poission $\left(\frac{D}{2^{L}}\right)$, hence:

$$
\begin{gathered}
\operatorname{Pr}\left(Y_{i} \geq 2\right)=1-\operatorname{Pr}\left(Y_{i}=0\right)-\operatorname{Pr}\left(Y_{i}=1\right) \\
=1-e^{-\frac{D}{2^{L}}} \cdot\left(1+\frac{D}{2^{L+1}}\right),
\end{gathered}
$$

and thus:

$\mathbb{E}(Y)=2^{L} \cdot\left(1-e^{-\frac{D}{2^{L}}} \cdot\left(1+\frac{D}{2^{L}}\right)\right)$.

Since $e^{-x} \cdot(1+x)>1-\frac{x^{2}}{2}$ for $0<x \leq 1$, we get

$$
\mathbb{E}(Y)<2^{L} \cdot\left(1-\left(1-\frac{D^{2}}{2^{2 L+1}}\right)\right)=\frac{D^{2}}{2^{L+1}} .
$$

Recall that $2^{L}=W \varepsilon^{-1}$ and $D \leq W$ and thus $\mathbb{E}(Y)<\frac{D \cdot \varepsilon}{2}$.

Note also that the $\left\{\tilde{Y}_{i}\right\}$ are independent, since the $\left\{Y_{i}\right\}$ are independent, and as they are indicator (Bernoulli) variables, in particular they are Poisson trials. Therefore, we may use a Chernoff bound on $Y$ as it is the sum of independent Poisson trials. We use the following Chernoff bound to continue:

Lemma 5.8 (Theorem 4.4, page 64 of [35]). Let $X_{1}, \ldots, X_{n}$ be independent Poisson trials such that: $\operatorname{Pr}\left(X_{i}\right)=p_{i}$, and let $X=\sum_{i=1}^{n} X_{i}$, then for $R \geq 6 \mathbb{E}(X), \operatorname{Pr}(X \geq R) \leq 2^{-R}$.

Lemma 5.8 allows us to approach Theorem 5.9, which is the main result for DisTinCTLB and shows that it provides an $\epsilon, \delta$ approximation of $D$, which we can then use in Corollary 5.10 to show that it solves the count distinct problem.

Theorem 5.9. Let $\delta \leq \frac{1}{128}$. Then:

$$
\operatorname{Pr}\left(D-Z \geq \frac{1}{2} \varepsilon D \cdot \log \left(\frac{2}{\delta}\right)\right) \leq \delta
$$


Proof. For $\delta \leq \frac{1}{128}, \log \left(\frac{2}{\delta}\right) \geq 6$ and thus, as

$\log _{2}\left(\frac{2}{\delta}\right) \cdot \frac{1}{2} \varepsilon D \geq 6 \mathbb{E}(Y)$ we can use Lemma 5.8 to get that:

$$
\operatorname{Pr}\left(Y \geq \frac{1}{2} \varepsilon D \cdot \log _{2}\left(\frac{2}{\delta}\right)\right) \leq 2^{-(\varepsilon D) \log _{2}\left(\frac{2}{\delta}\right)} \leq 2^{-\log _{2}\left(\frac{2}{\delta}\right)} \leq \frac{\delta}{2} .
$$

As $X$ monotonically increases with $D$, we use Lemma 5.7 to conclude that $\operatorname{Pr}\left(X \geq \frac{1}{2} \varepsilon D \cdot \log _{2}\left(\frac{2}{\delta}\right)\right) \leq \delta$.

The following readily follows, as claimed.

Corollary 5.10. DisTinctLB solves the

$(W, \epsilon, \delta)$-Approximate Count Distinct problem, for $\varepsilon_{D}=\frac{1}{2} \varepsilon \cdot \log _{2}\left(\frac{2}{\delta}\right)$, and any $\delta \leq \frac{1}{128}$. That is, for such $\delta$ we get: $\operatorname{Pr}\left(Z \geq\left(1-\varepsilon_{D}\right) \cdot D\right) \geq 1-\delta$.

\subsubsection{Analysis of DistinctMLE}

We now provide an analysis of the DisTinCTMLE estimation method, which is more accurate but offers two sided error. Our goal is to derive confidence intervals around DisTinCTMLE and for this we require a better analysis of $Z$, which is provided by Lemma 5.11 .

\section{Lemma 5.11.}

$$
D-\left(\begin{array}{c}
D \\
2
\end{array}\right) \cdot 2^{-L} \leq \mathbb{E}(Z) \leq D-\left(\begin{array}{c}
D \\
2
\end{array}\right) \cdot 2^{-L}+\left(\begin{array}{c}
D \\
3
\end{array}\right) \cdot 2^{-2 L} .
$$

Proof. This follows simply from 1 by expanding the binomial.

We also need to analyze the second moment of $Z$. Lemma 5.12 does just that.

\section{Lemma 5.12 .}

$$
\mathbb{E}\left(Z^{2}\right)=2^{2 L}-\left(2^{2 L+1}-2^{L}\right)\left(1-\frac{1}{2^{L}}\right)^{D}+\left(2^{2 L}-2^{L}\right)\left(1-\frac{1}{2^{L-1}}\right)^{D} .
$$

Proof. As in Theorem 5.6, we write $Z$ as a sum of indicator variables $Z=\sum_{i=0}^{2^{L}-1} Z_{i}$. Then

$$
Z^{2}=\sum_{i=0}^{2^{L}-1} Z_{i}^{2}+\sum_{i \neq j} Z_{i} Z_{j}
$$

By linearity of the expectation, it implies

$$
\mathbb{E}\left(Z^{2}\right)=\sum_{i=0}^{2^{L}-1} \mathbb{E}\left(Z_{i}^{2}\right)+\sum_{i \neq j} \mathbb{E}\left(Z_{i} Z_{j}\right)
$$

We note that for any $i \neq j,\left(1-Z_{i}\right)\left(1-Z_{j}\right)$ is also an indicator variable, attaining the value 1 with probability $\left(1-\frac{2}{2^{L}}\right)^{D}$. Therefore, for any $i \neq j$, we have by linearity of the expectation, that

$$
\left(1-\frac{2}{2^{L}}\right)^{D}=\mathbb{E}\left(1-Z_{i}\right)\left(1-Z_{j}\right)=1-\mathbb{E}\left(Z_{i}\right)-\mathbb{E}\left(Z_{j}\right)+\mathbb{E}\left(Z_{i} Z_{j}\right) .
$$

Since $\mathbb{E} Z_{i}=1-\left(1-\frac{1}{2^{L}}\right)^{D}$, it follows that

$$
\mathbb{E}\left(Z_{i} Z_{j}\right)=\left(1-\frac{2}{2^{L}}\right)^{D}+2 \cdot\left(1-\left(1-\frac{1}{2^{L}}\right)^{D}\right)-1=1+\left(1-\frac{2}{2^{L}}\right)^{D}-2\left(1-\frac{1}{2^{L}}\right)^{D} .
$$

Plugging it back in Equation 3, and using $Z_{i}^{2}=Z_{i}$, we obtain

$$
\mathbb{E}\left(Z^{2}\right)=2^{L} \cdot\left(1-\left(1-\frac{1}{2^{L}}\right)^{D}\right)+2^{L}\left(2^{L}-1\right)\left(1+\left(1-\frac{2}{2^{L}}\right)^{D}-2\left(1-\frac{1}{2^{L}}\right)^{D}\right) .
$$

Finally, expanding and rearranging we obtain the claim of this lemma. 
As our interest lies in the confidence intervals, we shall only need an approximation, described in the following simple corollary

Corollary 5.13. $D^{2}-\frac{4 \cdot D^{3}}{3 \cdot 2^{L}}<\mathbb{E}\left(Z^{2}\right)<D^{2}+\frac{D^{3}}{3 \cdot 2^{L}}$.

Proof. First, note that binomial expansion yields

$$
1-\frac{D}{2^{L}}+\frac{\left(\begin{array}{l}
D \\
2
\end{array}\right)}{2^{2 L}}-\frac{\left(\begin{array}{l}
D \\
3
\end{array}\right)}{2^{3 L}} \leq\left(1-\frac{1}{2^{L}}\right)^{D} \leq 1-\frac{D}{2^{L}}+\frac{\left(\begin{array}{l}
D \\
2
\end{array}\right)}{2^{2 L}}
$$

Plugging it back into Lemma 5.12, and expanding we get on the one hand

$$
\begin{aligned}
\mathbb{E}\left(Z^{2}\right)>2^{2 L}-\left(2^{2 L+1}-2^{L}\right) & \left(1-\frac{D}{2^{L}}+\frac{\left(\begin{array}{c}
D \\
2
\end{array}\right)}{2^{2 L}}\right)+\left(2^{2 L}-2^{L}\right)\left(1-\frac{D}{2^{L-1}}+\frac{\left(\begin{array}{c}
D \\
2
\end{array}\right)}{2^{2 L-2}}-\frac{\left(\begin{array}{c}
D \\
3
\end{array}\right)}{2^{3 L-3}}\right)= \\
= & D+2 \cdot\left(\begin{array}{c}
D \\
2
\end{array}\right)-2^{-L} \cdot\left(\frac{3 D(D-1)}{2}+\frac{8 D(D-1)(D-2)}{6}\right)=D^{2}-\frac{D(D-1)(8 D-7)}{6 \cdot 2^{L}}
\end{aligned}
$$

which yields the lower bound. On the other hand, we have

$$
\begin{aligned}
\mathbb{E}\left(Z^{2}\right)<2^{2 L}-\left(2^{2 L+1}-2^{L}\right)\left(1-\frac{D}{2^{L}}+\frac{\left(\begin{array}{l}
D \\
2
\end{array}\right)}{2^{2 L}}-\frac{\left(\begin{array}{c}
D \\
3
\end{array}\right)}{2^{3 L-3}}\right)+\left(2^{2 L}-2^{L}\right)\left(1-\frac{D}{2^{L-1}}+\frac{\left(\begin{array}{c}
D \\
2
\end{array}\right)}{2^{2 L-2}}\right)= \\
=D+2 \cdot\left(\begin{array}{c}
D \\
2
\end{array}\right)+2^{-L} \cdot\left(-\frac{3 D(D-1)}{2}+\frac{D(D-1)(D-2)}{3}\right)=D^{2}+\frac{D(D-1)(2 D-13)}{6 \cdot 2^{L}},
\end{aligned}
$$

which yields the upper bound. Thus, we have established the corollary.

Using Corollary 5.13 and Lemma 5.11 we can finally get an estimate for $\mathbb{E}(\widehat{D})$, as described in the following theorem. This shows that $\widehat{D}$ is unbiased up to an $O\left(D \epsilon^{2}\right)$ additive factor.

Theorem 5.14.

$$
-D \cdot \varepsilon^{2} \cdot\left(\frac{2}{3}+\frac{\varepsilon}{2 W^{3}}\right)<\mathbb{E}(\widehat{D})-D<D \cdot \varepsilon^{2} \cdot\left(2+\frac{1}{3(1-\varepsilon)^{3}}\right) .
$$

Proof. We first note that for $x \in[0,1)$ one has

$$
x+\frac{x^{2}}{2} \leq-\ln (1-x) \leq x+\frac{x^{2}}{2}+\frac{x^{3}}{3(1-x)^{3}} .
$$

Therefore, we have

$$
\frac{Z}{2^{L}}+\frac{Z^{2}}{2^{2 L+1}} \leq-\ln \left(1-\frac{Z}{2^{L}}\right) \leq \frac{Z}{2^{L}}+\frac{Z^{2}}{2^{2 L+1}}+\frac{Z^{3}}{3 \cdot\left(2^{L}-Z\right)^{3}} .
$$

Since always $Z \leq D$, and $\frac{D}{2^{L}} \leq \frac{W}{2^{L}}=\varepsilon$, we can take expectation and obtain, by linearity and monotonicity of the expectation, that

$$
\frac{\mathbb{E}(Z)}{2^{L}}+\frac{\mathbb{E}\left(Z^{2}\right)}{2^{2 L+1}} \leq \mathbb{E}\left(-\ln \left(1-\frac{Z}{2^{L}}\right)\right) \leq \frac{\mathbb{E}(Z)}{2^{L}}+\frac{\mathbb{E}\left(Z^{2}\right)}{2^{2 L+1}}+\frac{D^{3}}{3 \cdot\left(2^{L}-D\right)^{3}}
$$

We can now substitute Lemma 5.11 and Corollary 5.13 to obtain

$$
\begin{aligned}
-\mathbb{E}\left(\ln \left(1-\frac{Z}{2^{L}}\right)\right) \leq \frac{D}{2^{L}}+\frac{D}{2^{2 L+1}}+\frac{D^{3}}{6 \cdot 2^{3 L}}+\frac{\left(\begin{array}{l}
D \\
3
\end{array}\right)}{2^{3 L}}+\frac{D^{3}}{3 \cdot\left(2^{L}-D\right)^{3}} & \\
& \leq \frac{D}{2^{L}}+\frac{D}{2^{2 L+1}}+D \cdot\left(\frac{2 W^{2}}{2^{3 L}}+\frac{W^{2}}{3(1-\varepsilon)^{3} \cdot 2^{3 L}}\right) .
\end{aligned}
$$


Recalling 5 we see also that $-\ln \left(1-\frac{1}{2^{L}}\right) \geq \frac{1}{2^{L}}+\frac{1}{2^{2 L+1}}$. Combining both inequalities, we get

$$
\mathbb{E}(\widehat{D}) \leq D+D \cdot\left(\frac{2 W^{2}}{2^{2 L}+2^{L-1}}+\frac{W^{2}}{3(1-\varepsilon)^{3} \cdot\left(2^{2 L}+2^{L-1}\right)}\right) \leq D+D \cdot\left(\frac{2 W^{2}}{2^{2 L}}+\frac{W^{2}}{3(1-\varepsilon)^{3} \cdot 2^{2 L}}\right) .
$$

Since $2^{L}=W \varepsilon^{-1}$, this gives us the upper bound. On the other hand, substituting Lemma 5.11 and Corollary 5.13 in the left inequality given in (6) we have also

$$
-\mathbb{E}\left(\ln \left(1-\frac{Z}{2^{L}}\right)\right) \geq \frac{D}{2^{L}}+\frac{D}{2^{2 L+1}}-\frac{4 D^{3}}{6 \cdot 2^{3 L}} .
$$

From (5) we see also that $-\ln \left(1-\frac{1}{2^{L}}\right) \leq \frac{1}{2^{L}}+\frac{1}{2^{2 L+1}}+\frac{1}{3 \cdot\left(2^{L}-1\right)^{3}}$. Combining both inequalities, we get

$$
\mathbb{E}(\widehat{D}) \geq D-\frac{4 D^{3}}{6 \cdot\left(2^{2 L}+2^{L-1}+3\right)}-\frac{8 D}{3 \cdot 2^{3 L}} \geq D-D \cdot\left(\frac{2 D^{2}}{3 \cdot 2^{2 L}}+\frac{8}{3 \cdot 2^{3 L}}\right) .
$$

Since $D \leq W$ and $2^{L}=W \varepsilon^{-1}$, this yields the lower bound, as claimed.

Next, we bound the error probability. This is done in the following theorem.

Theorem 5.15. Let $\delta \leq \frac{1}{128}$ and denote $\varepsilon_{D}=\frac{1}{2} \varepsilon \cdot \log _{2}\left(\frac{2}{\delta}\right)$. Then

$$
\operatorname{Pr}\left(\widehat{D} \leq D \cdot\left(1-\varepsilon_{D}\right)\right) \leq \delta
$$

Proof. We first note that

$$
\operatorname{Pr}\left(\frac{\ln \left(1-\frac{Z}{2^{L}}\right)}{\ln \left(1-\frac{1}{2^{L}}\right)} \leq a\right)=\operatorname{Pr}\left(Z \leq 2^{L}\left(1-\left(1-\frac{1}{2^{L}}\right)^{a}\right)\right) .
$$

Now, using Corollary 5.10, and the fact that

$$
2^{L}\left(1-\left(1-\frac{1}{2^{L}}\right)^{a}\right) \leq a
$$

the result immediately follows.

Theorem 5.15 shows the soundness of the DisTinCTMLE estimator by proving that it is at least as accurate as the DistinctLB estimator.

\subsection{Analysis of entropy estimation functionality}

We now turn to analyze the entropy estimation. Recall that SWAMP uses the estimator $\widehat{H}$. If we denote by $F$ the set of distinct fingerprints in the last $W$ elements, it is given by

$$
\widehat{H}=-\sum_{h \in F} \frac{n_{h}}{W} \log \left(\frac{n_{h}}{W}\right),
$$

where $n_{h}$ is the number of occurrences of fingerprint $h$ in the window of last $W$ elements. We begin by showing that $\mathbb{E}(\widehat{H})$ approximates $H$, where

$$
H=-\sum_{y \in D} \frac{f_{y}^{W}}{W} \log \left(\frac{f_{y}^{W}}{W}\right)
$$

is the entropy in the window.

Theorem 5.16. $\widehat{H} \leq H$ and $\mathbb{E}(\widehat{H})$ is at least $H-\varepsilon$. 
Proof. For any $y \in D$, let $h(y)$ be its fingerprint. Recall that $\widehat{f_{y}^{W}}$ is the frequency $n_{h(y)}$ of $h(y)$, hence we have:

$$
\widehat{f_{y}^{W}}=\sum_{y^{\prime}: h(y)=h\left(y^{\prime}\right)} f_{y^{\prime}}^{W} \text {. }
$$

For ease of notation, we denote $p_{y}=\frac{f_{y}^{W}}{W}$, and $p_{h}=\frac{n_{h}}{W}$. Thus

$$
p_{h}=\sum_{y \in D: h(y)=h} p_{y} .
$$

It follows that:

$$
\widehat{H}=-\sum_{h \in F} p_{h} \log \left(p_{h}\right)=-\sum_{h \in F}\left(\sum_{y \in D: h(y)=h} p_{y}\right) \log \left(\sum_{y \in D: h(y)=h} p_{y}\right)=-\sum_{y \in D} p_{y} \cdot \log \left(\sum_{y^{\prime} \in D: h\left(y^{\prime}\right)=h(y)} p_{y^{\prime}}\right) .
$$

Now, since $p_{y^{\prime}} \geq 0$ for all $y^{\prime}$, it follows that for any $y$,

$$
\sum_{y^{\prime} \in D: h\left(y^{\prime}\right)=h(y)} p_{y^{\prime}} \geq p_{y} .
$$

Hence, by the monotonicity of the logarithm,

$$
\widehat{H} \leq-\sum_{y \in D} p_{y} \log \left(p_{y}\right)=H
$$

proving the first part of our claim. Conversely, denote for any $y \in D$ and any $h \in F$, by $I_{y, h}$ the Bernoulli random variable, attaining the value 1 if $h(y)=h$. Then we see that:

$$
\widehat{H}=-\sum_{y \in D} p_{y} \cdot \log \left(\sum_{y^{\prime} \in D} p_{y^{\prime}} \cdot I_{y^{\prime}, h(y)}\right) .
$$

We see, by using Jensen's inequality, the concavity of the logarithm and the linearity of expectation, that for any $y$ :

$$
\mathbb{E} \log \left(\sum_{y^{\prime} \in D} p_{y^{\prime}} \cdot I_{y^{\prime}, h(y)}\right) \leq \log \left(\sum_{y^{\prime} \in D} p_{y^{\prime}} \cdot \mathbb{E}\left(I_{y^{\prime}, h(y)}\right)\right)
$$

Since for any $y^{\prime} \neq y$, we have $\mathbb{E}\left(I_{y^{\prime}, h(y)}\right)=2^{-L}$, we see that

$$
\mathbb{E} \log \left(\sum_{y^{\prime} \in D} p_{y^{\prime}} \cdot I_{y^{\prime}, h(y)}\right) \leq \log \left(2^{-L} \cdot \sum_{y^{\prime} \neq y \in D} p_{y^{\prime}}+p_{y}\right)=\log \left(p_{y}+2^{-L}\left(1-p_{y}\right)\right)
$$

Summing this over $y$, and using the linearity of expectation once more, we obtain:

$$
\mathbb{E}(\widehat{H}) \geq-\sum_{y \in D} p_{y} \log \left(p_{y}+2^{-L}\left(1-p_{y}\right)\right)
$$

Subtracting $H$ yields:

$$
\mathbb{E}(\widehat{H})-H \geq-\sum_{y \in D} p_{y}\left(\log \left(p_{y}+2^{-L}\left(1-p_{y}\right)\right)-\log \left(p_{y}\right)\right) \geq-\sum_{y \in D} p_{y}\left(\log \left(1+2^{-L}\left(\frac{1}{p_{y}}-1\right)\right)\right) .
$$

Note that $2^{-L}=\frac{\varepsilon}{W}$ and $\frac{1}{W} \leq p_{y}<1$. Hence, $0<2^{-L}\left(\frac{1}{p_{y}}-1\right)<\varepsilon$. Since for any $0<x, \log (1+x)<x$, we get:

$$
\mathbb{E}(\widehat{H})-H \geq-\sum_{y \in D} 2^{-L} \cdot\left(1-p_{y}\right)=-\frac{\varepsilon}{W} \cdot(D-1) .
$$

As $D \leq W$, we get that $\mathbb{E}(\widehat{H})>H-\varepsilon$, as claimed. 
We proceed with a confidence interval derivation:

Theorem 5.17. Let $\epsilon_{H}=\varepsilon \delta^{-1}$ then $\widehat{H}$ solves $\left(W, \epsilon_{H}, \delta\right)$-Entropy Estimation. That is: $\operatorname{Pr}\left(H-\widehat{H} \geq \epsilon_{H}\right) \leq \delta$.

Proof. Denote by $X \triangleq H-\widehat{H}$ the random variable of the estimation error. According to Theorem 5.16, $X$ is non-negative and $E(X) \leq \epsilon$. Therefore, according to Markov's theorem we have

$$
\operatorname{Pr}\left(H \leq \widehat{H}+\epsilon \delta^{-1}\right)=1-\operatorname{Pr}\left(X \geq \epsilon \delta^{-1}\right) \geq 1-\frac{E(X)}{\epsilon \delta^{-1}} \geq 1-\delta . \square
$$

Setting $\epsilon_{H}=\epsilon \delta^{-1}$ and rearranging the expression completes the proof.

\section{Discussion}

In modern networks, operators are likely to require multiple measurement types. To that end, this work suggests SWAMP, a unified algorithm that monitors four common measurement metrics in constant time and compact space. Specifically, SWAMP approximates the following metrics on a sliding window: Bloom filters, per-flow counting, count distinct and entropy estimation. For all problems, we proved formal accuracy guarantees and demonstrated them on real Internet traces.

Despite being a general algorithm, SWAMP advances the state of the art for all these problems. For sliding Bloom filters, we showed that SWAMP is memory succinct for constant false positive rates and that it reduces the required space by 25\%-40\% compared to previous approaches [33]. In per-flow counting, our algorithm outperforms WCSS [3] - a state of the art window algorithm. When compared with $1+\varepsilon$ approximation count distinct algorithms [9, 22], SWAMP asymptotically improves the query time from $O\left(\varepsilon^{-2}\right)$ to a constant. It is also up to x1000 times more accurate on real packet traces. For the entropy estimation on a sliding window [23], SWAMP reduces the update time to a constant.

While SWAMP benefits from the compactness of TinyTable [17], most of its space reductions inherently come from using fingerprints rather than sketches. For example, all existing count distinct and entropy algorithms require $\Omega\left(\epsilon^{-2}\right)$ space for computing a $1+\epsilon$ approximation. SWAMP can compute the exact answers using $O(W \log W)$ bits. Thus, for a small $\epsilon$ value, we get an asymptotic reduction by storing the fingerprints on any compact table.

\section{References}

[1] Mohammad Alizadeh, Tom Edsall, Sarang Dharmapurikar, Ramanan Vaidyanathan, Kevin Chu, Andy Fingerhut, Vinh The Lam, Francis Matus, Rong Pan, Navindra Yadav, and George Varghese. CONGA: Distributed Congestion-aware Load Balancing for Datacenters. In ACM SIGCOMM, 2014.

[2] Ziv Bar-Yossef, T. S. Jayram, Ravi Kumar, D. Sivakumar, and Luca Trevisan. Counting distinct elements in a data stream. In RANDOM, 2002.

[3] Ran Ben-Basat, Gil Einziger, Roy Friedman, and Yaron Kassner. Heavy Hitters in Streams and Sliding Windows. In IEEE INFOCOM, 2016.

[4] Theophilus Benson, Aditya Akella, and David A. Maltz. Network traffic characteristics of data centers in the wild. In ACM IMC 2010, pages 267-280.

[5] Giuseppe Bianchi, Nico d'Heureuse, and Saverio Niccolini. On-demand time-decaying bloom filters for telemarketer detection. ACM SIGCOMM CCR, 2011.

[6] Burton H. Bloom. Space/time trade-offs in hash coding with allowable errors. Commun. ACM, 1970.

[7] Flavio Bonomi, Michael Mitzenmacher, Rina Panigrahy, Sushil Singh, and George Varghese. An improved construction for counting bloom filters. In ESA, 2006. 
[8] Vladimir Braverman, Rafail Ostrovsky, and Carlo Zaniolo. Optimal sampling from sliding windows. In $A C M$ PODS, 2009.

[9] Y. Chabchoub and G. Hebrail. Sliding hyperloglog: Estimating cardinality in a data stream over a sliding window. In IEEE ICDM Workshops, 2010.

[10] Yousra Chabchoub, Raja Chiky, and Betul Dogan. How can sliding hyperloglog and ewma detect port scan attacks in ip traffic? EURASIP Journal on Information Security, 2014.

[11] Philippe Chassaing and Lucas Gerin. Efficient estimation of the cardinality of large data sets. In DMTCS, 2006.

[12] Saar Cohen and Yossi Matias. Spectral bloom filters. In Proc. of the 2003 ACM SIGMOD Int. Conf. on Management of Data, 2003.

[13] Andrew R. Curtis, Jeffrey C. Mogul, Jean Tourrilhes, Praveen Yalagandula, Puneet Sharma, and Sujata Banerjee. DevoFlow: Scaling Flow Management for High-performance Networks. In ACM SIGCOMM, 2011.

[14] Nick Duffield, Carsten Lund, and Mikkel Thorup. Priority sampling for estimation of arbitrary subset sums. J. $A C M, 54(6)$.

[15] Marianne Durand and Philippe Flajolet. Loglog counting of large cardinalities. In ESA, 2003.

[16] G. Einziger and R. Friedman. TinyLFU: A highly efficient cache admission policy. In Euromicro PDP, 2014.

[17] Gil Einziger and Roy Friedman. Counting with TinyTable: Every Bit Counts! In ACM ICDCN, 2016.

[18] Gil Einziger and Roy Friedman. Tinyset - an access efficient self adjusting bloom filter construction. IEEE/ACM Transactions on Networking, 2017.

[19] Cristian Estan, George Varghese, and Mike Fisk. Bitmap algorithms for counting active flows on high speed links. In $A C M I M C, 2003$.

[20] Philippe Flajolet and G. Nigel Martin. Probabilistic counting algorithms for data base applications. $J$. Comput. Syst. Sci., 1985.

[21] Philippe Flajolet, ric Fusy, Olivier Gandouet, and et al. Hyperloglog: The analysis of a near-optimal cardinality estimation algorithm. In AOFA, 2007.

[22] Eric Fusy and Frcric Giroire. Estimating the number of Active Flows in a Data Stream over a Sliding Window, pages 223-231. 2007.

[23] Moshe Gabel, Daniel Keren, and Assaf Schuster. Anarchists, unite: Practical entropy approximation for distributed streams. In $K D D, 2017$.

[24] Pedro Garcia-Teodoro, Jesus E. Diaz-Verdejo, Gabriel Macia-Fernandez, and E. Vazquez. Anomaly-Based Network Intrusion Detection: Techniques, Systems and Challenges. Computers and Security, 2009.

[25] Frdric Giroire. Order statistics and estimating cardinalities of massive data sets. Discrete Applied Mathematics, 2009.

[26] Sudipto Guha, Andrew McGregor, and Suresh Venkatasubramanian. Streaming and sublinear approximation of entropy and information distances. In ACM SODA, pages 733-742, 2006.

[27] Stefan Heule, Marc Nunkesser, and Alexander Hall. Hyperloglog in practice: Algorithmic engineering of a state of the art cardinality estimation algorithm. In ACM EDBT, 2013.

[28] Paul Hick. CAIDA Anonymized Internet Trace, equinix-sanjose 2013-12-19 13:00-13:05 UTC, Direction B., 2014. 
[29] Paul Hick. CAIDA Anonymized Internet Trace, equinix-chicago 2016-02-18 13:00-13:05 UTC, Direction A., 2016.

[30] Nan Hua, Haiquan (Chuck) Zhao, Bill Lin, and Jun Xu. Rank-indexed hashing: A compact construction of bloom filters and variants. In ICNP. IEEE, 2008.

[31] Abdul Kabbani, Mohammad Alizadeh, Masato Yasuda, Rong Pan, and Balaji Prabhakar. AF-QCN: Approximate Fairness with Quantized Congestion Notification for Multi-tenanted Data Centers. In IEEE HOTI, 2010 .

[32] John Kubiatowicz, David Bindel, Yan Chen, Steven Czerwinski, Patrick Eaton, Dennis Geels, Ramakrishan Gummadi, Sean Rhea, Hakim Weatherspoon, Westley Weimer, Chris Wells, and Ben Zhao. Oceanstore: An architecture for global-scale persistent storage. SIGPLAN Not., 35(11), November 2000.

[33] Yang Liu, Wenji Chen, and Yong Guan. Near-optimal approximate membership query over time-decaying windows. In IEEE INFOCOM, 2013.

[34] Yi Lu, Andrea Montanari, Balaji Prabhakar, Sarang Dharmapurikar, and Abdul Kabbani. Counter braids: a novel counter architecture for per-flow measurement. In ACM SIGMETRICS, 2008.

[35] Michael Mitzenmacher and Eli Upfal. Probability and Computing: Randomized Algorithms and Probabilistic Analysis. Cambridge University Press, New York, NY, USA, 2005.

[36] Moni Naor and Eylon Yogev. Sliding Bloom Filter. In ISAAC, 2013.

[37] George Nychis, Vyas Sekar, David G. Andersen, Hyong Kim, and Hui Zhang. An empirical evaluation of entropy-based traffic anomaly detection. In $A C M I M C, 2008$.

[38] Prashant Pandey, Michael A. Bender, Rob Johnson, and Rob Patro. A general-purpose counting filter: Making every bit count. In ACM SIGMOD, 2017.

[39] Ori Rottenstreich, Yossi Kanizo, and Isaac Keslassy. The variable-increment counting bloom filter. In INFOCOM, 2012.

[40] MyungKeun Yoon. Aging bloom filter with two active buffers for dynamic sets. IEEE Trans. on Knowl. and Data Eng., 2010.

[41] L. Zhang and Y. Guan. Detecting click fraud in pay-per-click streams of online advertising networks. In ICDCS, 2008. 